\title{
Operation of gas electron multipliers in pure xenon at low temperatures
}

\author{
V. Solovov ${ }^{\mathrm{a}, *}$, F. Balau ${ }^{\mathrm{a}, \mathrm{b}}$, F. Neves ${ }^{\mathrm{a}}$, V. Chepel ${ }^{\mathrm{a}, \mathrm{b}}$, A. Pereira ${ }^{\mathrm{a}}$, M.I. Lopes ${ }^{\mathrm{a}, \mathrm{b}}$ \\ ${ }^{a}$ Laboratório de Instrumentação e Fisica Experimental de Particulas, LIP-Coimbra, Rua Larga, P-3004-516 Coimbra, Portugal \\ ${ }^{\mathrm{b}}$ CFRM of the Department of Physics of the University of Coimbra, 3004-516 Coimbra, Portugal
}

Available online 18 May 2007

\begin{abstract}
Operation of Gas Electron Multiplier (GEM) in ultra-pure xenon was studied for gas at $25^{\circ} \mathrm{C}$, cold but not saturated gas at $-90{ }^{\circ} \mathrm{C}$ and saturated xenon vapour at $-102^{\circ} \mathrm{C}$. The most stable operation was observed at $-90^{\circ} \mathrm{C}$ for which the maximum visible gain of 150 was obtained from a single GEM. The maximum gain of $\approx 40$ was achieved for saturated xenon vapour at $-102^{\circ} \mathrm{C}$ and of $\approx 25$ for twophase configuration at the same temperature. Continuous operation of a GEM in two-phase mode for periods of several hours was routinely achieved.
\end{abstract}

(C) 2007 Elsevier B.V. All rights reserved.

PACS: 29.40.Cs

Keywords: Xenon; Two-phase detectors; Gas electron muliplier; GEM

\section{Introduction}

Two-phase xenon (gas/liquid) detectors are being developed and used for several applications such as dark matter searches [1], neutrino detection [2] and medical imaging [3]. Detection of low-energy signals in those detectors requires internal amplification of the signal in the detector. The existing designs rely mostly on the production of electroluminescence in the gas by electrons extracted from the liquid.

An alternative approach is to multiply the extracted electrons in saturated vapour by means of a micro-pattern detector, such as Gas Electron Multiplier (GEM) [4]. Recently, successful operation of a triple-GEM structure in a two-phase xenon detector with gain of 200 was reported [5]. However, resolution was poor due to insufficient temperature stabilization and purity of the liquid.

We present the results on GEM operation in pure xenon gas at room temperature, at $-90^{\circ} \mathrm{C}$ and in saturated vapour at $-102^{\circ} \mathrm{C}$, including the double phase mode, in a well-controlled environment and at a constant gas density.

\footnotetext{
*Corresponding author. Tel.: + 351239410684 ; fax: + 351239822358.

E-mail address: solovov@lipc.fis.uc.pt (V. Solovov).
}

\section{Experimental set-up and results}

\subsection{Gas}

The measurements were carried out using the gridded ionisation chamber shown in Fig. 1. The stainless steel cathode with an ${ }^{241} \mathrm{Am} \alpha$-source mounted on top of it was separated from the Frisch grid by ceramic spacers. A $50 \mu \mathrm{m}$ thick GEM foil was placed between the Frisch grid and the collector grid. The GEM, manufactured by $3 \mathrm{M}$ [6], has holes of $70 \mu \mathrm{m}$ in diameter at $140 \mu \mathrm{m}$ pitch. Electrons from $\alpha$-tracks were drifted to the bottom face of the GEM, kept at ground potential, by applying negative voltage to the cathode plate, high enough to ensure full electron extraction from the $\alpha$-tracks. The Frisch grid was kept at a constant fraction of the cathode voltage by means of a voltage divider (see Fig. 2) to ensure constant transparency for drifting electrons of $\approx 95 \%$. Low-noise charge-sensitive preamplifiers (Cremat-110) were connected to the bottom face of the GEM and to the collector grid. The output signals of the preamplifiers were amplified and shaped by a spectroscopy amplifier (Canberra 2021) and analysed by a multi-channel analyzer.

Two modes of operation were used. In the first mode, the top face of the GEM and the collector grid were grounded. 
In this case all the charge was collected at the bottom face of the GEM. This allowed to measure the initial charge $\left(Q_{0}\right)$ arriving to the GEM. In the second mode, the initial charge was amplified in the GEM and partially extracted to the collector grid $\left(Q_{\text {ext }}\right)$ by applying positive voltage to the GEM and collector grid. The amplification factor $Q_{\text {ext }} / Q_{0}$ was recorded as a function of the voltage applied to the GEM $\left(V_{\mathrm{GEM}}\right)$ and the strength of the extraction field.

The electrodes were mounted on a stainless steel flange with ceramic feedthroughs and put inside a cylindrical

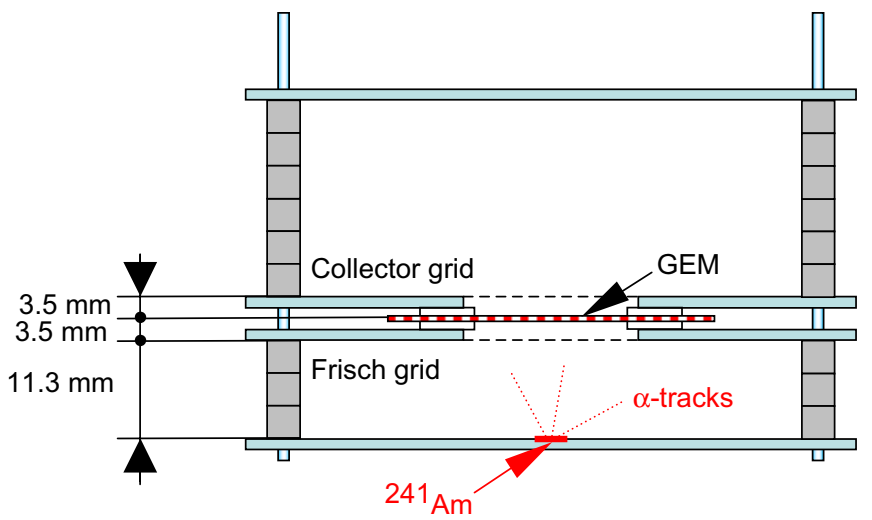

Fig. 1. The ionisation chamber used in the measurements $(5.49 \mathrm{MeV}$ $\alpha$-particles, emitted by ${ }^{241} \mathrm{Am}$, have range of $11 \mathrm{~mm}$ in Xe gas with density $4.9 \times 10^{19} \mathrm{~cm}^{-3}$ ). stainless steel chamber, $100 \mathrm{~mm}$ diameter and $70 \mathrm{~mm}$ high. Only high-vacuum compatible materials were used. The chamber was helium leak tested, pumped to $10^{-7} \mathrm{mbar}$ and baked at $75^{\circ} \mathrm{C}$ for three days. Then it was further cleaned by passage of xenon gas purified by Oxisorb column.

The chamber was placed inside a liquid nitrogen cryostat. Special care was taken to ensure temperature stability and uniformity along the chamber. By using copper screens around the chamber it was possible to maintain the temperature gradient between the top and the bottom of the chamber, as well as variations of the temperature during the measurements, within $2{ }^{\circ} \mathrm{C}$. The temperature was measured with precision of $0.5^{\circ} \mathrm{C}$ by means of three platinum thermo-resistors distributed along the body of the chamber.

Operation of a single GEM in xenon gas was studied at two gas densities (see Table 1). When determining the maximum amplification, the point was considered valid if it was possible to record a pulse height spectrum during at least one minute without discharges.

The results of measurements in gas are presented in Fig. 3 for gas density of $4.9 \times 10^{19} \mathrm{~cm}^{-3}$. The solid and the open symbols correspond to temperatures of 25 and $-90^{\circ} \mathrm{C}$, respectively. The amplification is similar for the two temperatures, but the overall stability of operation is better at lower temperature, allowing extraction of much higher fraction of the multiplied charge from the GEM by applying higher extraction field. The data regarding the

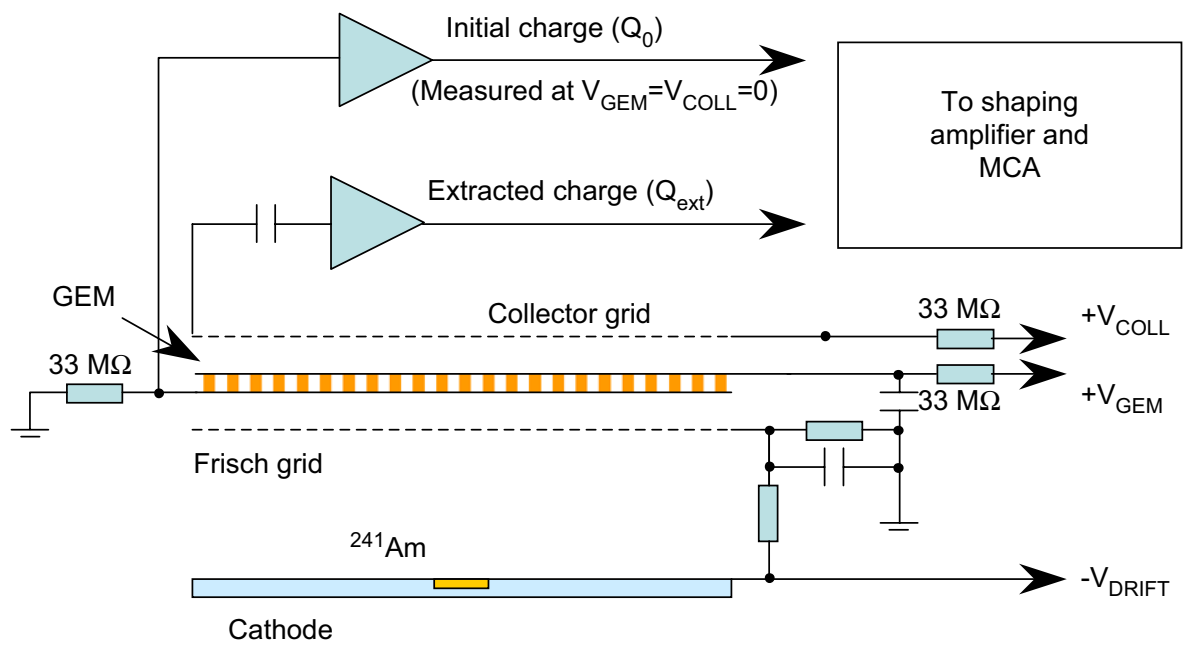

Fig. 2. The readout schematic.

Table 1

Maximum amplification factor reached at different conditions and corresponding $V_{\mathrm{GEM}}$

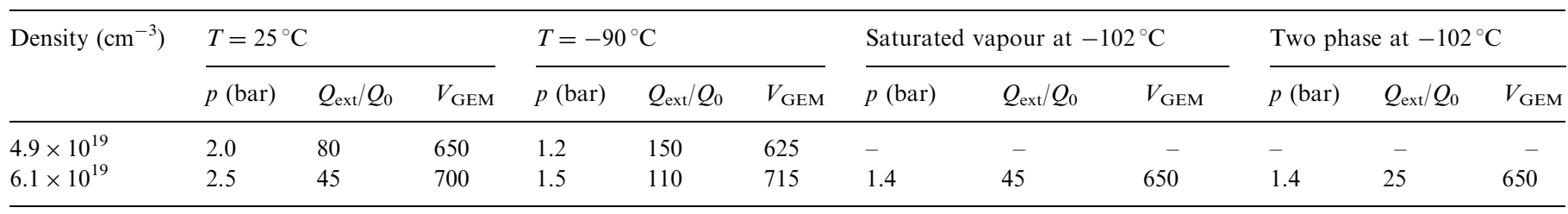




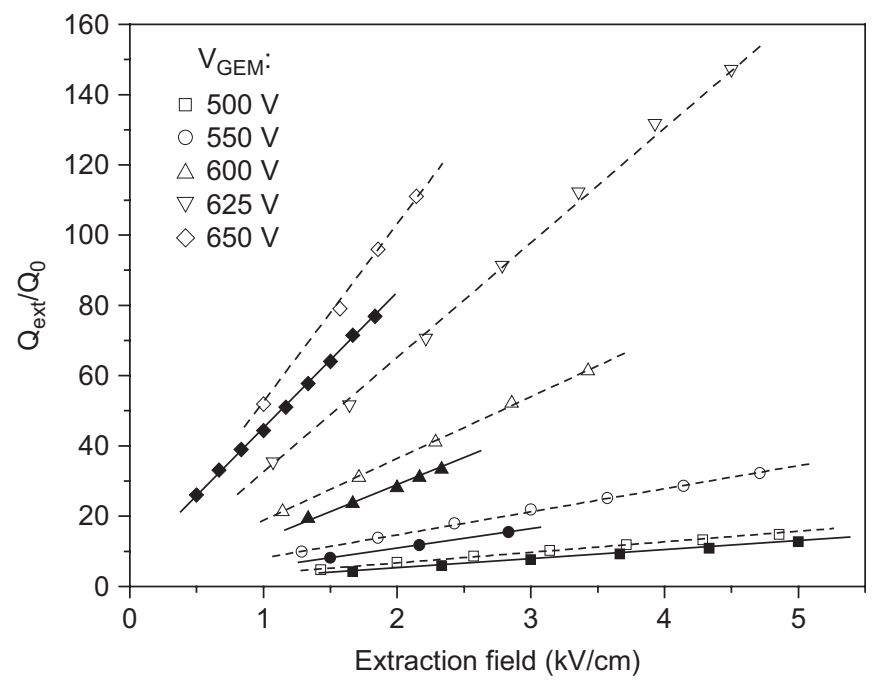

Fig. 3. Dependence of amplification $Q_{\text {ext }} / Q_{0}$ in xenon gas on the extraction field for several values of $V_{\mathrm{GEM}}$. Solid and open symbols correspond to $25^{\circ} \mathrm{C}$ and $-90^{\circ} \mathrm{C}$, respectively. Gas density is $4.9 \times 10^{19} \mathrm{~cm}^{-3}$.

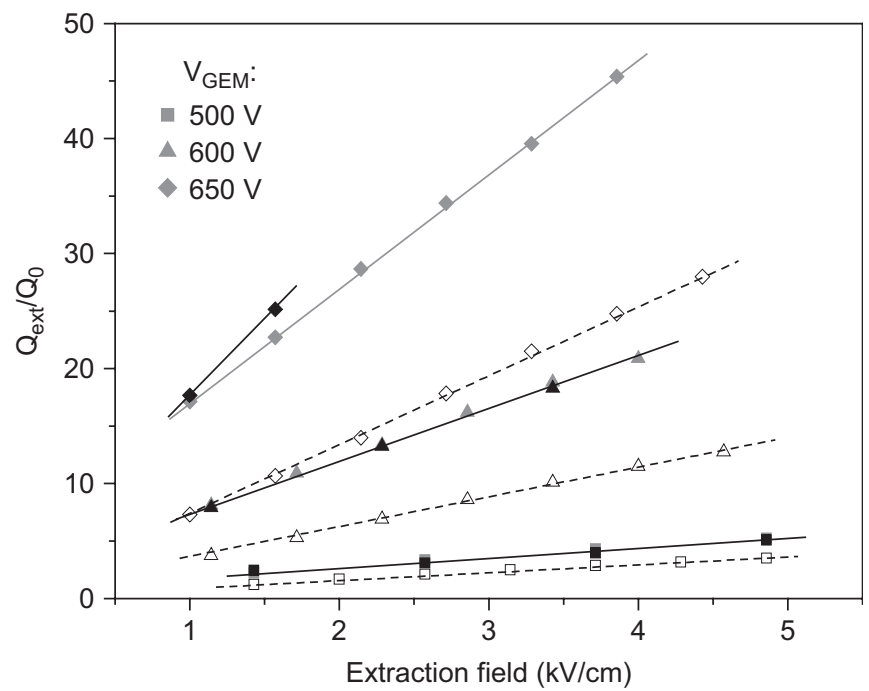

Fig. 4. Dependence of amplification $Q_{\text {ext }} / Q_{0}$ on the extraction field for several values of $V_{\mathrm{GEM}}$ at $\rho=6.1 \times 10^{19} \mathrm{~cm}^{-3}$. Gray symbols-saturated gas, black symbols - two phase mode, open symbols - gas at $-90^{\circ} \mathrm{C}$.

maximum amplification reached at different conditions are summarized in Table 1.

\subsection{Saturated vapour and two-phase mode}

The measurements were also performed for saturated xenon vapour at $-102^{\circ} \mathrm{C}$. To do this, xenon was condensed into the chamber until the liquid level was about $1 \mathrm{~mm}$ below the cathode. Then the temperature was stabilized until the gradient along the chamber was less then $1{ }^{\circ} \mathrm{C}$. It was found that the operation of the GEM in saturated xenon vapour differs considerably from operation in cold gas of the same density, showing higher gain at the same voltage (Fig. 4). This is probably due to higher concentration of dimers $\mathrm{Xe}_{2}$ with lower ionization potential. However, the stability in vapour is lower and the maximum amplification of only 45 was reached for vapour at $-102{ }^{\circ} \mathrm{C}$, compared to $Q_{\text {ext }} / Q_{0} \approx 110$ obtained in gas at $-90{ }^{\circ} \mathrm{C}$.

In the two-phase mode, the electrons extracted from a particle track in the liquid phase must be transported to the liquid-gas interface and "pushed" into the gas phase by a strong electric field [7]. To provide the conditions for efficient and uniform emission of electrons into the gas, the chamber was slightly modified. In particular, the Frisch grid was removed in order to ensure better field uniformity at the surface of the liquid and the distance between the cathode and the GEM was reduced to $11.0 \mathrm{~mm}$.

The chamber was filled with liquid xenon up to a level of $7 \mathrm{~mm}$ above the $\alpha$-source. The voltage applied to the cathode was increased to $6 \mathrm{kV}$ resulting in a field of $\approx 4 \mathrm{kV} /$ $\mathrm{cm}$ in the liquid, at which about $80 \%$ of electrons reaching the liquid-gas boundary are emitted into the gas phase [8]. It is worth mentioning that due to strong recombination, the charge that is extracted from an $\alpha$-track in the liquid by a field of $4 \mathrm{kV} / \mathrm{cm}$ is only about $5 \%$ of that in the gas [9]. In our case, the total charge transported to the GEM at these conditions was found to be $2.7 \mathrm{fC}$ per $\alpha$-particle.

The dependence of amplification on $V_{\mathrm{GEM}}$ and the extraction field in two-phase mode is practically identical to that observed for saturated vapour (Fig. 4). The pulse height spectrum of signals taken from the collector grid at $V_{\mathrm{GEM}}=625 \mathrm{~V}$ and $E_{\mathrm{ext}}=5.7 \mathrm{kV} / \mathrm{cm}$ is shown in Fig. 5 . The amplification factor is about 15 at these conditions. For the peak on the right, which is due to $\alpha$-particles, the FWHM of $16 \%$ is found. The right side of the peak seems to be distorted, most probably due to simultaneous detection of $60 \mathrm{keV} \gamma$-rays also emitted from the ${ }^{241} \mathrm{Am}$ source. Fitting of the left side of the peak with a Gaussian gives resolution of $13.5 \%$ (FWHM). The smaller peak at channel 70 seems to be due to $60 \mathrm{keV} \gamma$-rays, for which,

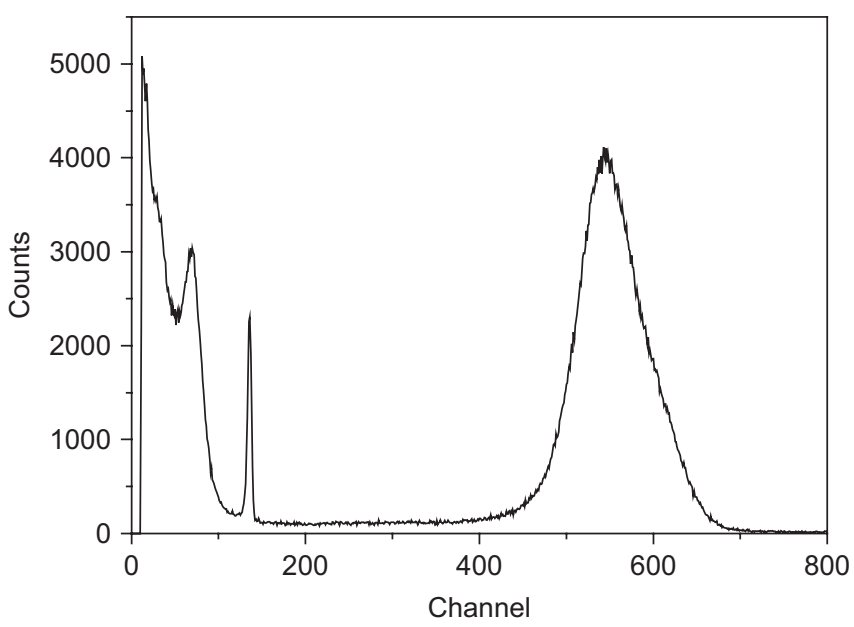

Fig. 5. Pulse-height spectrum obtained in the two-phase mode. $V_{\mathrm{GEM}}=625 \mathrm{~V}, Q_{\mathrm{ext}} / Q_{0}=15$. Narrow peak at channel 130 is due to calibration pulses. 
because of much weaker recombination, the charge extraction in liquid is much more efficient than for $\alpha$ particles.

\subsection{Xenon purity}

The issue of possible contamination of liquid xenon by electronegative impurities from GEM is very important as impurities may affect both the validity of the measurements and performance of the detector. To assess the purity of xenon after a 12-h run, additional xenon was condensed into the chamber to cover the GEM (the amount of xenon added was about $10 \%$ of xenon already existing in the chamber). Then the time dependence of the current, induced by ionisation charge drifting in the parallel-plate chamber formed by the bottom face of the GEM and the cathode was studied. As this current was found practically constant during the electron drift time of about $3.5 \mu \mathrm{s}$, thus indicating no sensible loss in the amount of drifting electrons during this time, the electron lifetime is much longer than $3.5 \mu \mathrm{s}$. Also, the total charge collected from $\alpha$-track was estimated to be $3.2 \mathrm{fC}$ for electric field of $5.5 \mathrm{kV} / \mathrm{cm}$, which is in agreement with published data [9] within $10 \%$.

\section{Conclusions}

Operation of a single GEM in pure xenon was studied for gas at $25^{\circ} \mathrm{C}$, cold but not saturated gas at $-90{ }^{\circ} \mathrm{C}$ and saturated xenon vapour in one- and two-phase modes. The most stable operation was observed at $-90{ }^{\circ} \mathrm{C}$ for which the gain of 150 was obtained for xenon gas at a density of $4.9 \times 10^{19} \mathrm{~cm}^{-3}$. The maximum gain of $\approx 40$ was achieved for saturated xenon vapour at $-102{ }^{\circ} \mathrm{C}$ and of $\approx 25$ for two-phase configuration at the same temperature. Energy resolution of $13.5 \%$ (FWHM) was obtained for $\alpha$-particles in two-phase mode. Continuous stable operation of a GEM in two-phase mode for periods of several hours was routinely achieved.

\section{Acknowledgements}

This work was supported by Fundação para a Ciência e Tecnologia, Portugal (project POCTI/FP/63446/2005) and INTAS (project INTAS 04-78-6744). V. Solovov was receiving a post-doctoral fellowship (SFRH/BPD/14517/ 2003) from the same institution.

\section{References}

[1] D.Yu. Akimov, et al., Astropart. Phys. 27 (2007) 46.

[2] C. Hagmann, A. Bernstein, IEEE Trans. Nucl. Sci. NS-51 (2004) 2151.

[3] V. Egorov, et al., Nucl. Instr. and Meth. 205 (1983) 373.

[4] F. Sauli, Nucl. Instr. and Meth. A 386 (1997) 531.

[5] A. Bondar, et al., Nucl. Instr. and Meth. A 556 (2006) 273.

[6] P.S. Barbeau, et al., Nucl. Instr. and Meth. A 515 (2003) 439.

[7] A.I. Bolozdynya, et al., IEEE Trans. Nucl. Sci. NS-42 (1995) 565.

[8] E.M. Gushchin, et al., Sov. Phys. JETP 55 (1982) 650.

[9] E. Aprile, et al., Nucl. Instr. and Meth. A 307 (1991) 119. 